\title{
First Trimester Screening for Common Trisomies and Microdeletion 22q11.2 Syndrome Using Cell-Free DNA: A Prospective Clinical Study
}

\author{
Karl Oliver Kagan ${ }^{a}$ Markus Hoopmann ${ }^{a}$ Theresa Pfaffa Natalia Prodan ${ }^{a}$ \\ Philipp Wagner $^{a}$ Maximilian Schmid ${ }^{b}$ Andreas Dufke ${ }^{c} \quad$ Ulrike Mau-Holzmann ${ }^{c}$ \\ Sara Brucker $^{a}$ Livia Marcato ${ }^{d}$ Barbara Malvestiti ${ }^{d}$ Francesca Romana Grati ${ }^{d}$ \\ aDepartment of Women's Health, University Women's Hospital, Tuebingen, Germany; ${ }^{b}$ Roche Sequencing Solutions \\ Inc., Ariosa Diagnostics Inc., San Jose, CA, USA; Institute of Medical Genetics and Applied Genomics, University

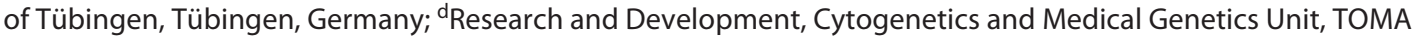 \\ Advanced Biomedical Assays S.p.A., Impact Lab Group, Busto Arsizio, Italy
}

\section{Keywords}

Microdeletion 22q11.2 - Aneuploidy · Screening · Genetics ·

Pregnancy

\begin{abstract}
Objectives: The aims of the study were to assess the falsepositive and uninformative test rate with first trimester cellfree DNA (cfDNA) screening for common trisomies and microdeletion 22q11.2 (22q11.2DS) and to examine women's attitudes toward such an approach. Methods: This is a prospective study at the Prenatal Medicine Department of the University of Tübingen, Germany, at 11-13 weeks. In all pregnancies, a detailed ultrasound examination was carried out, followed by a cfDNA analysis for common trisomies and 22q11.2DS. In cases where the cfDNA analysis indicated 22q11.2DS, invasive prenatal diagnostic testing and parental testing were performed. After delivery, a detailed neonatal clinical examination was carried out including further genetic testing. Prior to counselling about the study, we asked the pregnant women who were potentially eligible for the study to anonymously report on their knowledge about 22q11.2DS. Results: A total of 1,127 pregnancies were included in the final analysis of the study. The first cfDNA test was uninformative in 15 (1.33\%) pregnancies. In 10 (0.89\%)
\end{abstract}

cases, the test remained uninformative, even after the second blood sample. There were $3(0.27 \%)$ cases with a positive cfDNA test for 22q11.2DS. In all, 983 women returned the anonymous questionnaire prior to study participation. Only $80(8.1 \%)$ women responded that they felt familiar or very familiar with 22q11.2DS. Conclusion: The addition of 22q11.2DS in first trimester cfDNA screening for common trisomies is feasible. The uninformative test rate for common trisomies and 22 q $11.2 \mathrm{DS}$ is $0.9 \%$, and the false-positive rate for $22 q 11.2 D S$ is $0.3 \%$. Awareness and education around 22q11.2DS should be improved.

(c) 2020 S. Karger AG, Basel

\section{Introduction}

For the last 2 decades, first trimester combined screening (FTCS) has been considered the gold standard in screening for trisomies 21, 18, and 13 [1]. However, this approach has been challenged by the introduction of cellfree DNA (cfDNA) screening. It is likely that this method will replace classical FTS as the primary test for detection

Trial registration no: NCT03375359.

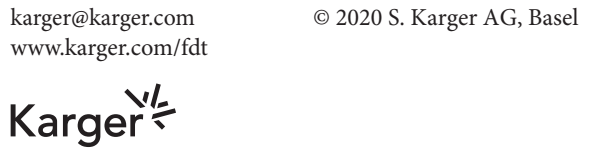




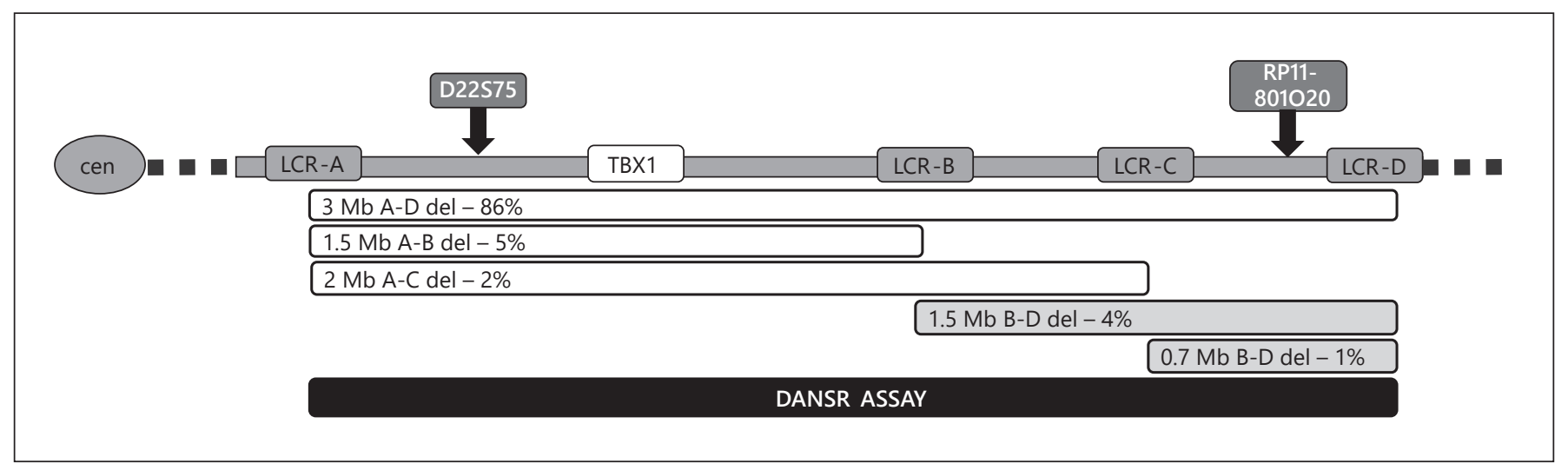

Fig. 1. Schematic representation of the structure of the 22q11.2DS critical region. White bars represent proximal deletions and gray bars, central deletions. The percentage represents the proportion of 22q11.2DS patients with each specific type of deletion. The black bar represents the microarray probe coverage of the DANSR targeted assay. The position of the FISH probes (D22S75 and RP11-801O20) are also shown. DANSR, Digital ANalysis of Selected Regions; FISH, fluorescence in situ hybridization; LCR, low-copy repeat.

of common trisomies [2]. The detection rate for each of the common trisomies is about $99 \%$ for a false-positive rate of $<0.1 \%$ [3].

Current research in cfDNA technology focuses on the detection of other chromosomal conditions, especially the 22q11.2 microdeletion syndrome (22q11.2DS; MIM\# 188400) [4]. This autosomal dominant disease is caused by different microdeletions that are mediated by low-copy repeats (LCRs; LCR22A $\rightarrow$ LCR22H) mapping to the q11.2 region of chromosome 22 (Fig. 1). The first 4 LCRs (LCR-A $\rightarrow$ LCR-D) account for the majority of 22 q11.2DS cases. Over $90 \%$ of patients have "proximal" deletions encompassing the LCR22A-D (A-D, A-C, and $A-B)$. About $5 \%$ of patients have "central" deletions encompassing B-D LCRs. Distal D-H deletions are rare [5, 6].

The gold standard test for prenatal diagnosis of detecting 22q11.2DS is chromosomal microarray (CMA) performed on genomic DNA from invasively obtained samples (chorionic villi and amniotic fluid) [4]. There are also targeted assays available (i.e., Prenatal BACs-on-Beads, fluorescence in situ hybridization [FISH], and multiplex ligation-dependent probe amplification [MLPA]), which are often utilized in familial cases where the microdeletion has already been identified with these technologies in the proband [4].

Both genome-wide and targeted cfDNA test methodologies can be used to screen for 22q11.2DS [4]. Targeted technologies are designed to interrogate the proximal deletions (A-B, A-C, A-D, A-E, or A-F) at a high analysis depth. Nontargeted genome-wide technologies based on massively parallel shotgun sequencing (MPSS) by a shallow NGS of maternal and fetal circulating cfDNA fragments include the whole chromosome 22. Screening for the entire A-H region is technically feasible through a bioinformatics post hoc process extrapolating from chromosome 22 only the selected fragments of interest from the $\mathrm{q} 11.2$ region [4].

Microdeletion 22q11.2 has a heterogenous clinical presentation with multi-organ dysfunction. Common postnatal phenotypic findings include growth and developmental delay, cardiac defects, cleft palate, recognizable facial features, learning disabilities, and immunodeficiency [7-10]. Overall, it is the second most common cause of developmental delay and major congenital heart disease after Down syndrome [11]. Despite the full penetrance, it shows a highly variable expressivity so that there are affected individuals with milder phenotypes that may remain unrecognized, even by healthcare professionals. Antenatally, the diagnosis is generally prompted in cases with a fetal anomaly, especially a cardiac defect [12].

In prenatal samples, the prevalence of the disease is dependent on the ultrasound findings. Fetuses with congenital heart defects show the highest prevalence, while in anatomically normal fetuses with a normal karyotype, it is in the range of 1:800-1:1,000. The prevalence is independent on maternal age $[4,13-16]$.

So far, most studies on cfDNA screening for 22q11.2DS have either used artificial or biobank samples or, when testing clinical populations, provide incomplete outcome data $[17,18]$. In a large retrospective study from Martin et al. [17], the authors reviewed the 22q11.2DS 
results with the targeted SNP-based cfDNA technology in a general population. The positive predictive value (PPV) was $15.7 \%$. Only one large prospective study of general population screening has been published so far [19]. Using an MPSS-based cfDNA test applied in 94,085 second trimester pregnancies, they reported a false-positive rate of $0.001 \%(0-0.006)$, a PPV of $92.9 \%$ (68.5$98.7)$, and a sensitivity of $86.7 \%$ (62.2-96.3) based on the clinical examination at birth and molecular follow-up of low-risk cases with fetal/newborn anomalies. Due to the lack of prospective screening studies and clinical validation, most scientific societies do not endorse routine cfDNA screening for 22q11.2DS. Herein, we present a prospective clinical study, aimed at assessing the uninformative test rate and the false-positive rate with first trimester cfDNA screening for common trisomies and 22q11.2DS and examining women's attitudes toward such an approach.

\section{Methods}

This is a prospective study, which was performed at the Prenatal Medicine Department of the University of Tübingen, Germany. The subjects were pregnant women who were referred for first trimester risk assessment. In our department, first trimester risk assessment is routinely done at 11-13 weeks' gestation. In addition to crown-rump length and nuchal translucency (NT) measurements, it also includes a detailed ultrasound examination based on ISUOG guidelines [20]. All operators who perform this examination are certified by the FMF UK.

In general, we do not calculate a specific risk for aneuploidy if the NT measurement is $>3.5 \mathrm{~mm}$ or if a fetal anomaly is identified. These cases are deemed to be at a very high risk for the whole spectrum of chromosomal abnormalities and are offered invasive testing.

If the NT thickness is $3.5 \mathrm{~mm}$ or less, we offer cfDNA testing to assess the risk for trisomies 21, 18, and 13. Assessment of sex chromosome aneuploidy is not offered.

For the purpose of this study, the cfDNA analysis was performed by TOMA Advanced Biomedical Assays S.p.A., Impact Lab Group (Busto Arsizio, VA, Italy) using Harmony ${ }^{\circledR}$ Prenatal Test (Roche, Inc., San Jose, CA, USA) as previously described [21]. In brief, this is a targeted cfDNA analysis performed using microarray quantitation of DANSR (Digital ANalysis of Selected Regions) assays of non-polymorphic (chromosomes 13, 18, 21, X, and $\mathrm{Y}$ ) and polymorphic loci (chromosomes 1-12) to estimate chromosome proportion and fetal fraction, respectively [22]. The FORTE (Fetal-fraction Optimized Risk of Trisomy Evaluation) algorithm is used to provide patient-specific risk assessments for trisomy [23].

Approximately 500 additional DANSR assays were designed against targets uniformly distributed within a $3.0-\mathrm{Mb}$ region of 22q11.2 between LCR-A and D (Fig. 1). Custom DNA microarrays were manufactured by Affymetrix Inc. (Santa Clara, CA, USA) to specifically quantify all DANSR assay products. Each sample was analyzed on a single custom microarray [18].

Prospective Screening for Microdeletion

22q11.2 Syndrome
In all cases where a cfDNA analysis is performed, an additional tube of blood is collected for backup FTCS in case of an uninformative cfDNA testing result, which is expected in a certain proportion of cases. This serum sample is centrifuged at 4,000 rpm for $5 \mathrm{~min}$; the serum is aliquoted and stored at $-80^{\circ} \mathrm{C}$. In any case where no result is obtained by cfDNA analysis, the serum sample is gently thawed and free beta-hCG and PAPP-A levels are measured. The risk for trisomy 21 is then computed using FTCS according to the algorithm of the FMF UK [1].

All women with pregnancies who were referred to us during the study period were considered eligible for this study. The only exclusion criteria were maternal age $<18$ years, CRL measurement of $>84$ or $<45 \mathrm{~mm}$, and multiple pregnancy, including vanishing twins.

We included women who were referred to us for routine FTCS and those who were sent with abnormal FTCS results. In the latter group, the risk was predominantly increased due to increased NT. As increased NT is also a marker for cardiac defects, we suspected a higher risk for fetuses with 22q11.2DS in this group. Therefore, for the purpose of this study, cfDNA screening for 22q11.2DS was performed in all cases, irrespective of the NT thickness and of whether there was a fetal defect or not.

In cases where the cfDNA analysis indicated a high probability of 22q11.2DS, invasive diagnostic testing by amniocentesis or chorionic villus sampling (CVS) was carried out. Parental testing was carried out on peripheral T-lymphocytes. Standard karyotyping was performed following national cytogenetic and molecular cytogenetic guidelines [24]. In general, chromosomes were prepared from cultured cells according to standard procedures and characterized by molecular karyotyping and/or standard banding. The number of analyzed metaphases was a minimum of 15 per sample at a 400 GTG-band resolution level or higher and at least 10 metaphases per FISH probe. For CMA analysis, genomic DNA was isolated by routine procedures.

For 22q11.2DS assessment in cases with a positive cfDNA test result, the analysis was conducted by CMA on DNA from fresh CVS biopsy or long-term culture in combination with FISH analysis on spontaneous metaphases from cytotrophoblasts using BAC probes mapping in the proximal (A-B LCRs; D22S75 [Abbott/Vysis]) and in the central critical regions (C-D LCRs; RP11-801O20 provided by Thomas Liehr, Jena, Germany). FISH analysis was performed when CMA provided a normal result to exclude the presence of rare confined placental mosaicism for 22q11.2DS in cytotrophoblasts [25]. Parental testing was carried out by FISH analysis. After delivery, a detailed neonatal clinical examination was performed, including further genetic testing on cord blood or placenta by FISH and/or microarray.

High-resolution chromosome analysis (SNP array analysis) was performed using the CytoScan ${ }^{\mathrm{TM}}$ Optima Array (Thermo Fisher Scientific, Waltham, MA USA) according to the manufacturer's instructions. Genotypes were called with Chromosome Analysis Software (version 3.0.0.139). The minimum detection limit for copy number variants was $50 \mathrm{~kb}$ with at least 5 markers included. All nucleotide positions refer to the Human Genome February 2009 assembly (GRCh37/hg19). We filtered for quality parameters (median absolute pairwise difference $\leq 0.25$ and waviness standard deviation $\leq 0.12$ ).

In pregnancies with a normal cfDNA test result for 22q11.2DS, further genetic examinations were not routinely carried out. However, in Germany, all children are examined directly after birth and at least 6 times by a pediatrician within the first year of life. At the 
Table 1. Summary of the study characteristics

\begin{tabular}{lcc}
\hline & High risk, $n=65$ & Low risk, $n=1,062$ \\
\hline Maternal age, years, median (25-75th centile) & $35.8(30.4-38.3)$ & $33.9(31.0-36.7)$ \\
Gestational age, weeks, median (25-75th centile) & $12.9(12.4-13.2)$ & $12.9(12.5-13.3)$ \\
Maternal weight, kg, median (25-75th centile) & $66.2(60.0-73.7)$ & $65.6(59.0-74.5)$ \\
Cigarette smoking, $n(\%)$ & $2(3.1)$ & $21(2.0)$ \\
Caucasian origin, $n(\%)$ & $64(98.5)$ & $1,027(97.3)$ \\
Assisted reproduction, $n(\%)$ & $4(6.2)$ & $35(3.3)$ \\
Crown-rump length, mm, median (25-75th centile) & $67.8(58.5-73.8)$ & $68.5(63.6-73.3)$ \\
NT thickness, median $(25-75$ th centile) & $3.7(1.9-5.0)$ & $1.8(1.6-2.1)$ \\
Fetal defects, $n(\%)$ & $44(67.9 \%)$ & 0 \\
\hline
\end{tabular}

NT, nuchal translucency.

Fig. 2. Description of the study population.

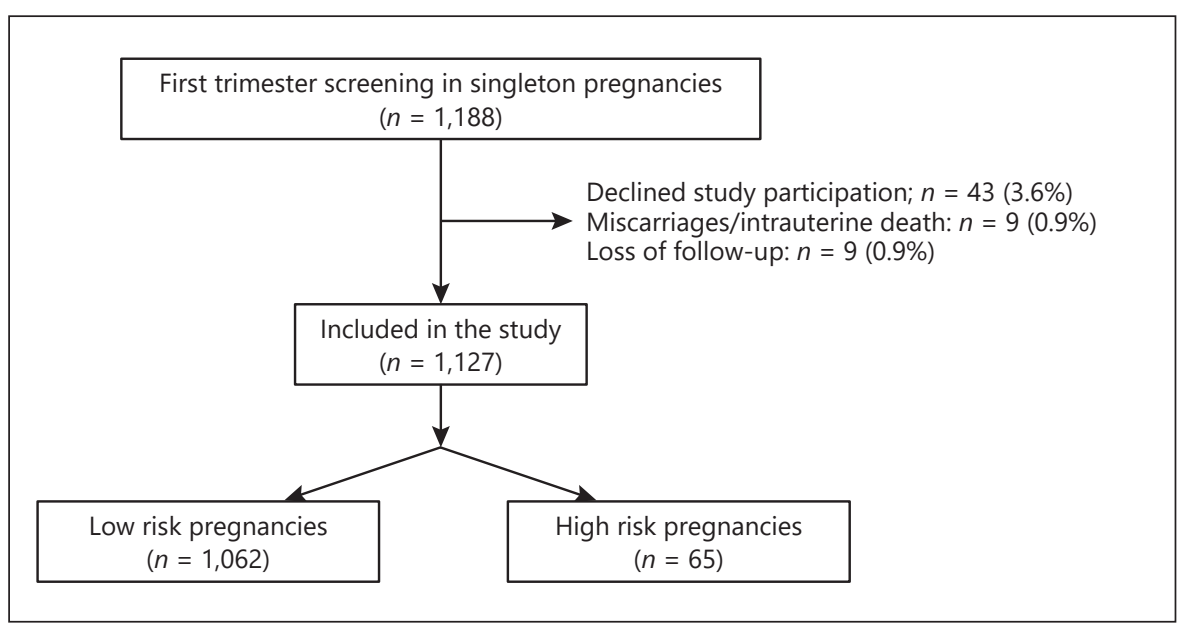

time of the manuscript preparation, the median age of the children was 354 days (range 198-542 days).

Clinically relevant ultrasound and screening information was recorded in the Viewpoint database (GE Healthcare, Munich, Germany). Information on maternal weight and height was extracted from the patient records. Ethnicity, smoking status, and mode of conception were assessed using a questionnaire, and this information was also recorded in the Viewpoint database. Outcome data were added as soon as it became available. The study was accompanied by a questionnaire to assess the women's knowledge about 22q11.2DS and their attitude toward screening.

Prior to counselling about the study and, in particular, about the spectrum of physical and mental development of children with 22q11.2DS, we asked the pregnant women who were potentially eligible for the study to anonymously report on their knowledge about 22q11.2DS. For comparison, they were also asked about the common trisomies; toxoplasmosis, parvovirus B19, and cytomegalovirus infection; and common pregnancy complications, namely, preeclampsia, gestational diabetes, and fetal growth restriction. The following answers were allowed: don't know at all, rather unknown, familiar, or very familiar. Four weeks after the final results of the cfDNA test were available, we asked the pregnant women (including those with false-positive results) again whether they find that an expansion of cfDNA testing to include $22 \mathrm{q} 11.2 \mathrm{DS}$ is useful (possible responses: not useful, rather not useful, useful, or very useful) and if they would recommend cfDNA screening in general and screening for 22q11.2DS in particular to their best friend (possible responses: no, rather no, rather yes, or yes). Approval for the study was obtained from the local Ethics Committee (University Hospital of Tübingen, Germany; October 18, 2017; No. 644/2017BO1). The study was registered in the clinicaltrial. gov registry (NCT03375359).

\section{Statistical Analysis}

The study cohort was divided into a high- and a low-risk group according to the presence of an increased NT of $>3.5 \mathrm{~mm}$ or of a fetal defect that requires postnatal follow-up. The additional ultrasound risk markers nasal bone as well as tricuspid and ductus venosus flow were not considered as anomalies.

Primary endpoints were the proportion of false-positive and uninformative cfDNA tests for 22q11.2DS. These results were stratified according to the 2 risk groups. The results of the questionnaires are given as proportion with 1 of the 2 positive answers. Results are given as median with the 25 and 75th interquartile range or as proportion with the 95th confidence interval using the Clopper-Pearson method. 
Table 2. Characteristics of the 3 cases with a false-positive cfDNA test result for microdeletion $22 \mathrm{q} 11.2$

\begin{tabular}{llll}
\hline Characteristic & Case 1 & Case 2 & Case 3 \\
\hline Maternal age, years & 35.2 & 41.3 & 29.7 \\
Gestational age, weeks & 12.4 & 13.4 & 12.9 \\
Maternal weight, kg & 63.5 & 129.0 & 56.8 \\
Ethnicity & Caucasian & Caucasian & Afro-Caribbean \\
Cigarette smoking & No & No & No \\
Assisted reproduction & No & No & No \\
Medical history & None & None & Sickle cell anemia \\
Crown-rump length, mm & 61.5 & 76.1 & 67.7 \\
NT, mm & 1.5 & 2.0 & 1.9 \\
NB/TF/DVF & $\mathrm{n} / \mathrm{n} / \mathrm{n}$ & $\mathrm{n} / \mathrm{n} / \mathrm{n}$ & $\mathrm{n} / \mathrm{n} / \mathrm{n}$ \\
Fetal defects & None & None & None \\
Free beta hCG in MoM & 1.19 & 1.74 & 0.60 \\
PAPP-A in MoM & 1.82 & 1.38 & 0.80 \\
Group & Low risk & Low risk & Low risk \\
Fetal fraction, \% & 9.0 & 7.0 & 6.0 \\
Pregnancy complications & Premature rupture of & None & Sickle cell crisis \\
Gestational age at delivery, weeks & membranes at 36 weeks & & 37.0 \\
Birth weight, g & 36.3 & 37.3 & 3,250 \\
\hline
\end{tabular}

NT, nuchal translucency; cfDNA, cell-free DNA.

\section{Results}

Between January and December 2018, 1,188 women with singleton pregnancies were referred for first trimester risk assessment. Of those, 43 (3.6\%) women declined study participation. Thus, 1,145 women initially participated in this study. However, $9(0.9 \%)$ pregnancies were lost to follow-up, and $9(0.9 \%)$ women miscarried the fetuses during the subsequent course of the pregnancy. In these pregnancies, the first trimester risk assessment or the cfDNA test was low risk and none opted for an autopsy or genetic testing. They were excluded from further analysis. Thus, 1,127 pregnancies were included in the final analysis (Fig. 2).

These pregnancies were divided into 2 groups according to the result of the ultrasound examination. The highrisk group consisted of 65 fetuses, either due to a fetal defect $(n=44)$ or due to an isolated increased NT thickness of $3.5 \mathrm{~mm}$ or more $(n=21)$. Within this group, 20 fetuses had trisomy 21,18 , or 13 .

The remaining 1,062 cases were classified as low-risk pregnancies. The characteristics of the study population are given in Table 1.

In the study population, there were 26 fetuses with trisomies 21,18 , and 13 . The results of the ultrasound examination are shown in online suppl. Table 1 (see www. karger.com/doi/10.1159/000510069 for all online suppl. material) as are the results of serum and additional ultra- sound markers. The cfDNA test was uninformative in 2 of these cases; both fetuses had trisomy 18. In all abnormal cases with a test result, the cfDNA test indicated a high-risk result. For trisomies 21,18 , and 13, there were no false-positive results.

In the whole study population, the first cfDNA test was uninformative in 15 (1.33\%, 95\% CI 0.75-2.19\%) pregnancies. In $10(0.89 \%)$ cases, the test remained uninformative, even after the second blood sample. Seven $(0.66 \%)$ of these pregnancies were in the low-risk group, and 3 $(4.6 \%)$ were in the high-risk group. In both groups, the median fetal fraction was $11.0 \%$. We did not observe cases that were partially uninformative. The median maternal weight of the women with a final uninformative test was $68.7 \mathrm{~kg}$. In this group, there was one neonate with a birth weight below the 5 th centile.

There were $3(0.27 \%, 95 \%$ CI $0-0.78 \%)$ cases with a positive cfDNA test for 22q11.2DS. All belonged to the low-risk group. The fetal fractions were 6.0, 7.0, and 9.0\%. The maternal and pregnancy characteristics of these 3 cases are highlighted in Table 2.

In all 3 cases, neither the pre- and postnatal testing (prenatal: 2 CVS, 1 amniotic fluid; postnatal: 1 fetal cord blood, 2 placental analyses) nor the parental testing indicated 22q11.2DS. Clinical examination by the pediatrician was also normal. In none of the children of the whole study population was there a suspicion of 22q11.2DS. 


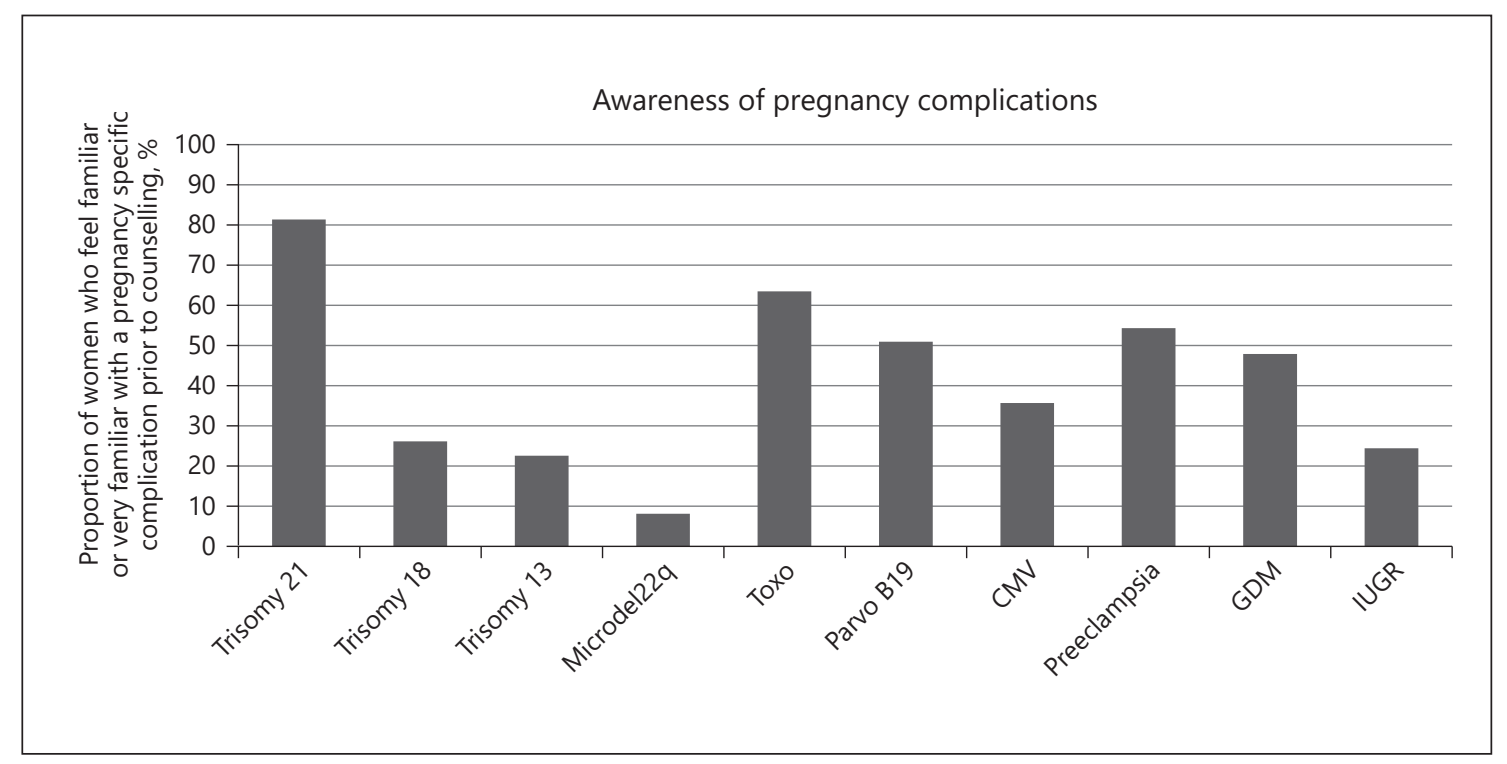

Fig. 3. Awareness of pregnancy complications.

Prior to the cfDNA study participation and the associated counselling, the pregnant women were asked if they knew anything about 22q11.2DS. For comparison, we also asked them about their knowledge of trisomies 21,18 , and 13 as well as in utero infections and common pregnancy complications. A total of 983 women returned the anonymous questionnaire. Only 80 (8.1\%, 95\% CI 6.5-10.3\%) women responded that they felt familiar or very familiar with 22q11.2DS. Their responses about the other pregnancy complications are shown in Figure 3. For none of the conditions was the maternal knowledge as low as for 22q11.2DS. The posttest questionnaire was returned by 1,057 pregnant women. In all, 1,013 (95.8\%, 95\% CI 94.5$97.0 \%$ ) women found that the expansion of cfDNA screening to include 22q11.2DS was useful or very useful; 1,046 (99.0\%, 95\% CI 98.2-99.5\%) women would recommend cfDNA screening to their best friend and 1,006 (95.2\%, 95\% CI 93.7-96.4\%) of them with the inclusion of 22q11.2DS. All 3 women with false-positive results for 22q11.2DS would still recommend cfDNA testing to their best friend and 2 women even with the inclusion of 22q11.2DS.

\section{Discussion}

In this prospective clinical study, we have demonstrated that cfDNA screening for common trisomies and $22 \mathrm{q} 11.2 \mathrm{DS}$ results in an uninformative test rate of $0.9 \%$ (none gave a partial uninformative result for 22q11.2DS only) and a false-positive rate for $22 \mathrm{q} 11.2 \mathrm{DS}$ of $0.3 \%$. In addition, we observed that the disease is rather unknown, as $<10 \%$ of the pregnant women feel familiar or very familiar with 22q11.2DS. However, after screening, about $95 \%$ of the women would recommend cfDNA screening with the inclusion of 22q11.2DS to their best friend. Our study was not designed to comment on the detection rate.

In a previous prospective study, we examined the test performance of cfDNA screening for trisomies 21,18 , and 13 without 22q11.2DS in fetuses without structural defects and a NT thickness of $<3.5 \mathrm{~mm}$. There were no false-positive cases, and the uninformative test rate was $1.5 \%$ using the same procedure as in this study [2]. Compared to our previous study with a similar design, the overall false-positive rate and uninformative test rate in this study were not increased by the additional screening for 22q11.2DS.

Screening for 22q11.2DS by cfDNA can be carried out by targeted and genome-wide technologies [4]. Still, for none of the technologies are enough clinical prospective data available so far. Table 3 summarizes the findings of the recent clinical studies.

Wapner et al. [26] used a SNP-based platform and examined 43 and 65 artificial samples from affected and unaffected pregnancies. They reported that all cases were correctly identified, and among the 3 real samples, 2 were detected [26]. Ravi et al. [27] also used a SNPbased technology with a revised protocol and detected 9 of 10 affected samples. There was one false-positive case 


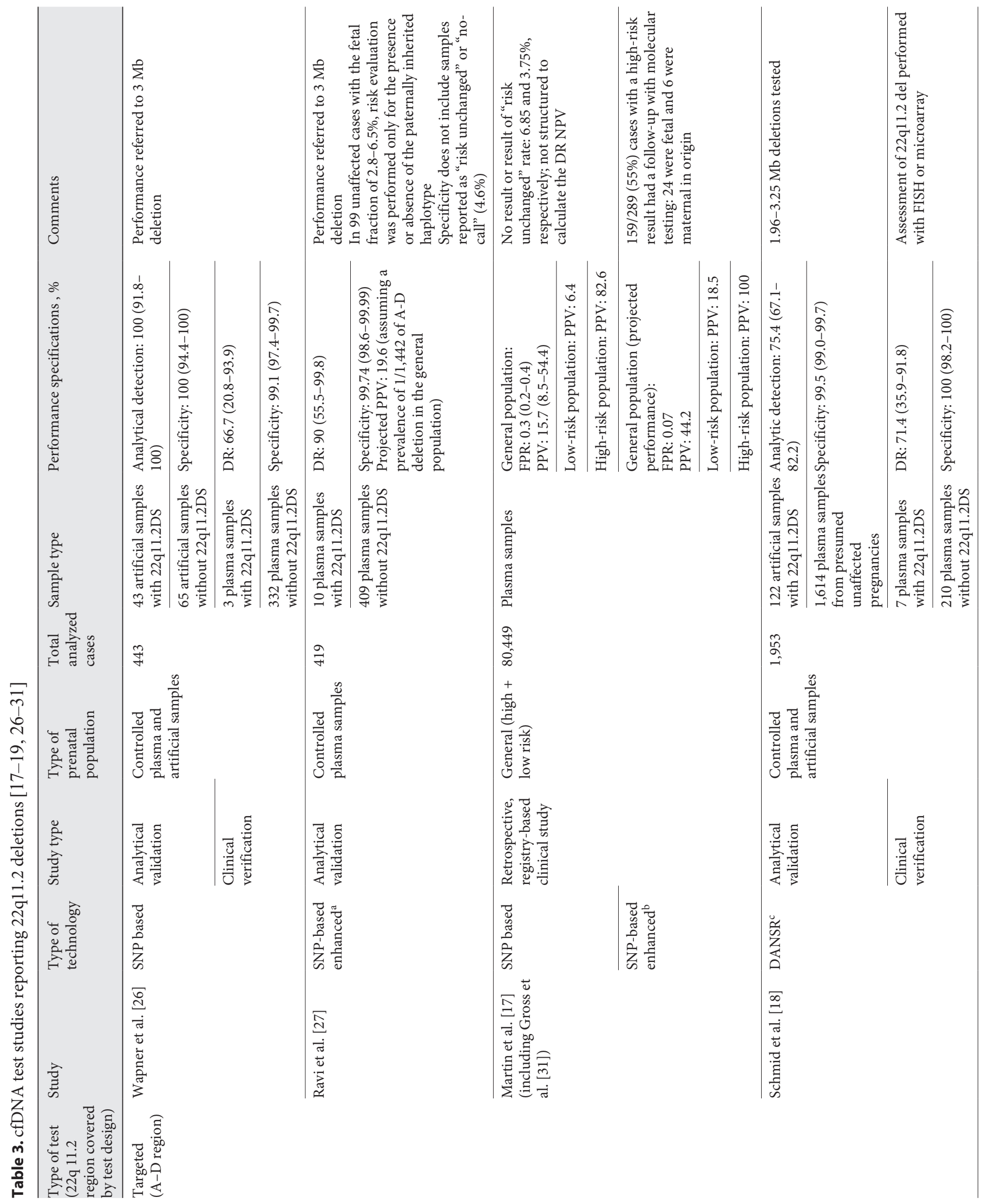




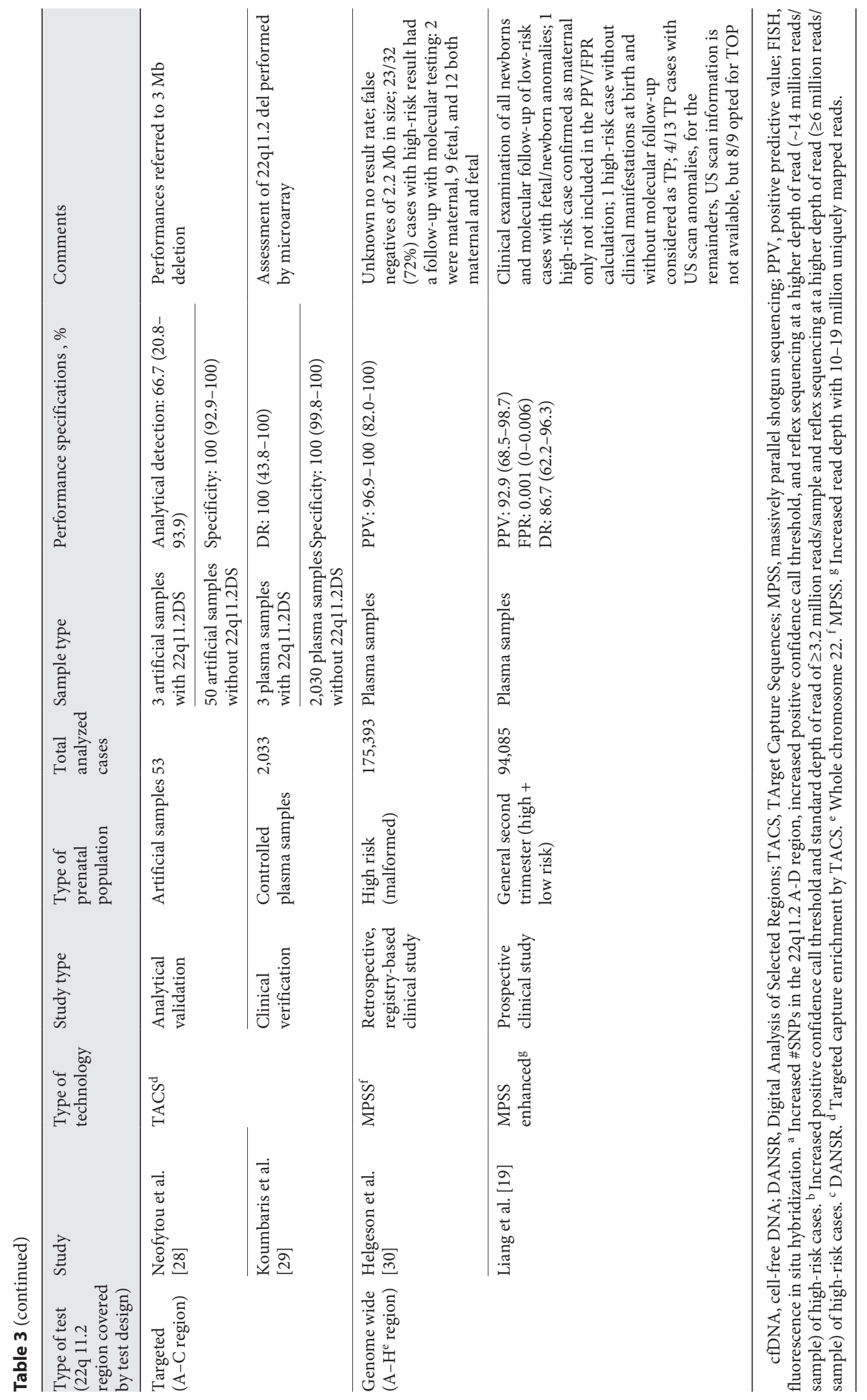


among 390 unaffected pregnancies [27]. In a large retrospective study including $>80,000$ samples, the false-positive rate of a SNP-based test was $0.1-0.3 \%$. In this study, reflex testing of high-risk 22q11.2DS cases with an increased sequencing depth decreased the false-positive rate to $0.07 \%$. Unfortunately, in the high-risk group, in about half of the cases (159/289), no outcome was available [17]. Within the latter group, there were 24 affected fetuses and 6 affected mothers [17]. Schmid et al. [18] used the DANSR assay probe design and examined 1,736 artificial and 217 real samples including 122 and 7 cases with 22 q11.2DS and 1,824 presumed or known unaffected samples and reported on a combined detection rate of $75.2 \%(67.1-81.8)$ and a specificity of $99.6 \%$ (99.1-99.8). Neofytou et al. [28] used a TACS-based assay and examined 3 and 50 artificial samples from affected and unaffected pregnancies. They reported that 2 of the 3 cases were correctly identified and no false-positive results. Koumbaris et al. [29] described the clinical verification with the same technology including 3 and 2,030 controlled plasma samples with and without 22q11.2DS, respectively. They reported a sensitivity and a specificity of $100 \%$ (43.8-100; 99.8-100, respectively). Helgeson et al. [30] retrospectively reviewed the results of $>175,000$ genome-wide examinations in high-risk pregnancies. There were 32 abnormal examinations for $22 \mathrm{q} 11.2 \mathrm{DS}$, and in 23 and 8 cases, the results were either confirmed in the mother or the fetus or at least clinically suspected. The incidence of fetal 22q11.2DS was threefold lower than with a SNP-based targeted technology $(0.01 \%, 24 / 80449$ vs. $0.03 \%, 21 / 175393)$, strongly suggesting under-ascertainment by MPSS [4]. Liang et al. [19] prospectively analyzed $>94,000$ second trimester pregnancies from the general population with a genome-wide test. They performed a clinical examination of all newborns, and low-risk cases with fetal/newborn anomalies underwent molecular follow-up. They reported a high sensitivity of $86.7 \%(62.2-96.3)$. The incidence of fetal 22q11.2DS is $1 / 7,200(13 / 94,000)$, which is far from the $1 / 1,000$ prevalence in an unselected prenatal population [16]. This supports the evidence that the high sensitivity might be inflated by undetected cases with subtle/absent clinical signs at birth. They also reported a false-positive rate of $0.001 \%(0-0.006)$ and a high PPV of 92.9 (68.5-98.7). In this population, pregnant women underwent a routine fetal ultrasound scan at 18 weeks' gestation. Four of 13 TP cases showed malformations compatible with 22q11.2DS; therefore, the inclusion of cases with US scan anomalies might have inflated the PPV.

Prospective Screening for Microdeletion

22q11.2 Syndrome
In summary, the results of previous studies are in line with our results. However, our study is the first to report on the false-positive rate and the test failure rate at 11-13 weeks in a prospective clinical study design with a detailed clinical follow-up of the entire study population, which included at least 6 pediatric examinations to investigate features consistent with 22q11.2DS.

We can only speculate about the origin of the 3 falsepositive results. We have used FISH and microarray analysis for pre- and postnatal follow-up testing and, in all cases, found normal results. In general, potential reasons for discordant results between the cfDNA analysis and the fetal genetic examination may be technical issues, maternal 22q11.2DS, feto-placental mosaicism, absence of heterozygosity, and a dichorionic twin pregnancy with a vanishing twin $[4,9,32]$. A recent study performing a systematic analysis of copy number variations associated with early pregnancy loss showed that 22q11.2DS was the submicroscopic imbalance most frequent in miscarriage. Therefore, it was considered to be associated with early pregnancy loss, possibly due to major cardiac malformations incompatible with pregnancy progression and development [33]. While vanishing twin was a reason for exclusion in our study, cases may be missed if the gestational sac can no longer be identified. Still, they can be the origin of discordant results [25].

Although from a technical standpoint it would be easy to add further conditions to the spectrum of cfDNA screening, scientific societies do not endorse antenatal cfDNA screening for 22q11.2DS so far. The International Society for Prenatal Diagnosis (ISPD) recommends that cfDNA screening should be limited to clinically significant disorders or well-defined severe conditions where the test performance of the screening test is well established [34]. Because 22q11.2DS shows variable expressivity, one could argue that the prenatal diagnosis may produce more harm than good as the severity of the phenotype in the newborn cannot be predicted. However, there are several potential benefits of antenatal screening for this condition. Despite the variable expressivity, the syndrome shows full penetrance, meaning that all 22q11.2DS patients have clinical manifestations. Currently, the majority of fetuses with 22q11.2DS are identified by genetic diagnosis after the detection of fetal anomalies, usually cardiac defects, in the second trimester or later. Screening for $22 \mathrm{q} 11.2 \mathrm{DS}$ in the first trimester would provide the possibility of anticipatory care and early diagnosis for neonatal complications $[7,9,35,36]$. The spectrum of 22q11.2DS involves more than antenatally detectable de- 
fects. For example, hypocalcemia and subsequent neonatal seizures, dysphagia, and immune defects will certainly remain undetected before birth and may require neonatal intervention [36,37]. As such, perinatal management and the clinical outcome can be significantly improved with the knowledge of the microdeletion and transition of care to a pediatric specialist. Although, to the best of our knowledge, there is no study comparing the outcome of children with 22q11.2DS with and without antenatal diagnosis, one can speculate that the knowledge of 22q11.2DS before birth would reduce the associated morbidity and mortality [9].

Clear strengths of our study are the prospective design, its initiation by a prenatal medicine team and not by a laboratory, the detailed follow-up examination of the screen-positive pregnancies, the high outcome rate of all screen-negative pregnancies, and the detailed ultrasound examination that was carried out prior to the screening test. The monocentric setup of our study can be considered as the most relevant weakness. Also, for the purpose of this study, we included high-risk pregnancies but grouped them separately.

\section{Conclusion}

In summary, the addition of 22q11.2DS to first trimester cfDNA screening for common trisomies with a targeted cfDNA approach is feasible and does not substantially increase the false-positive rate of the test. Awareness and education around the clinical relevance of 22q11.2DS should be improved.

\section{Statement of Ethics}

The research presented in the article was conducted ethically in accordance with the World Medical Association Declaration of Helsinki and the appropriate guidelines for human studies, as well as according to animal welfare regulations, including the Animal Research: Reporting of In Vivo Experiments (ARRIVE) guidelines, and was approved by the Ethics Committee at the University Hospital of Tübingen, Germany (October 18, 2017; No. 644/2017BO1). The study was registered in the clinicaltrial.gov registry (NCT03375359). Written consent was obtained from all patients who participated in the study.

\section{Conflict of Interest Statement}

Maximilian Schmid is an employee of Roche Sequencing Solutions Inc. Francesca Romana Grati, Livia Marcato, and Barbara Malvestiti are full-time employees of TOMA Laboratory without ownership shares. F.R. Grati is an expert panel member for Roche and Menarini Biomarkers. The unnamed authors have no disclosures.

\section{Funding Sources}

Ariosa has sponsored this study, which was set up as an investigator-initiated study (IIT with Karl Oliver Kagan as principal investigator).

\section{Author Contributions}

K.O.K.: conception, planning, carrying out, analyzing, and writing. M.H.: carrying out. T.P.: carrying out and analyzing. N.P.: carrying out. P.W.: carrying out. M.S.: planning. A.D.: planning, carrying out, and writing. U.M.H.: carrying out. S.B.: planning. L.M.: carrying out. B.M.: carrying out. F.R.G.: planning, carrying out, analyzing, and writing.

\section{References}

1 Santorum M, Wright D, Syngelaki A, Karagioti $\mathrm{N}$, Nicolaides $\mathrm{KH}$. Accuracy of first-trimester combined test in screening for trisomies 21, 18 and 13. Ultrasound Obstet Gynecol. 2017 Jun;49(6):714-20.

2 Kagan KO, Sroka F, Sonek J, Abele H, Lüthgens $\mathrm{K}$, Schmid M, et al. First-trimester risk assessment based on ultrasound and cell-free DNAvscombined screening: a randomized controlled trial. Ultrasound Obstet Gynecol. 2018 Apr;51(4):437-44.

3 Gil MM, Accurti V, Santacruz B, Plana MN, Nicolaides KH. Analysis of cell-free DNA in maternal blood in screening for aneuploidies: updated meta-analysis. Ultrasound Obstet Gynecol. 2017 Sep;50(3):302-14.
4 Grati FR, Gross SJ. Noninvasive screening by cell-free DNA for 22q11.2 deletion: benefits, limitations, and challenges. Prenat Diagn. 2019 Jan;39(2):70-80.

5 Morrow BE, McDonald-McGinn DM, Emanuel BS, Vermeesch JR, Scambler PJ. Molecular genetics of 22q11.2 deletion syndrome. Am J Med Genet A. 2018 Oct;176(10):2070-81.

6 Burnside RD. 22q11.21 deletion syndromes: a review of proximal, central, and distal deletions and their associated features. Cytogenet Genome Res. 2015;146(2):89-99.

7 McDonald-McGinn DM, Tonnesen MK, Laufer-Cahana A, Finucane B, Driscoll DA, Emanuel BS, et al. Phenotype of the 22q11.2 deletion in individuals identified through an affected relative: cast a wide FISHing net! Genet Med. 2001 Jan;3(1):23-9.
8 Kruszka P, Addissie YA, McGinn DE, Porras AR, Biggs E, Share M, et al. 22q11.2 deletion syndrome in diverse populations. Am J Med Genet A. 2017 Apr;173(4):879-88.

9 Dugoff L, Mennuti MT, McDonald-McGinn DM. The benefits and limitations of cell-free DNA screening for 22q11.2 deletion syndrome. Prenat Diagn. 2016 Jul 25;37(1):5360.

10 Meler E, Sisterna S, Borrell A. Genetic syndromes associated with isolated fetal growth restriction. Prenat Diagn. 2020;40(4):432-46.

11 Rauch A, Hoyer J, Guth S, Zweier C, Kraus C, Becker C, et al. Diagnostic yield of various genetic approaches in patients with unexplained developmental delay or mental retardation. Am J Med Genet A. 2006 Oct 1;140(19):206374. 
12 Schindewolf E, Khalek N, Johnson MP, Gebb J, Coleman B, Crowley TB, et al. Expanding the fetal phenotype: prenatal sonographic findings and perinatal outcomes in a cohort of patients with a confirmed 22q11.2 deletion syndrome. Am J Med Genet A. 2018 Aug; 176(8):1735-41.

13 Wang Y, Cao L, Liang D, Meng L, Wu Y, Qiao F, et al. Prenatal chromosomal microarray analysis in fetuses with congenital heart disease: a prospective cohort study. Am J Obstet Gynecol. 2018 Feb;218(2):244.e1-17.

14 Jansen FA, Blumenfeld YJ, Fisher A, Cobben JM, Odibo AO, Borrell A, et al. Array comparative genomic hybridization and fetal congenital heart defects: a systematic review and meta-analysis. Ultrasound Obstet Gynecol. 2015 Jan;45(1):27-35.

15 McDonald-McGinn DM, Sullivan KE, Marino B, Philip N, Swillen A, Vorstman JA, et al. 22q11.2 deletion syndrome. Nat Rev Dis Primers. 2015 Nov 19;1(1):15071-19.

16 Grati FR, Molina Gomes D, Ferreira JC, Dupont C, Alesi V, Gouas L, et al. Prevalence of recurrent pathogenic microdeletions and $\mathrm{mi}-$ croduplications in over 9500 pregnancies. Prenat Diagn. 2015 Aug;35(8):801-9.

17 Martin K, Iyengar S, Kalyan A, Lan C, Simon AL, Stosic M, et al. Clinical experience with a single-nucleotide polymorphism-based noninvasive prenatal test for five clinically significant microdeletions. Clin Genet. 2018 Feb; 93(2):293-300.

18 Schmid M, Wang E, Bogard PE, Bevilacqua E, Hacker C, Wang S, et al. Prenatal screening for 22q11.2 deletion using a targeted microarray-based cell-free DNA test. Fetal Diagn Ther. 2018;44(4):299-304.

19 Liang D, Cram DS, Tan H, Linpeng S, Liu Y, Sun $\mathrm{H}$, et al. Clinical utility of noninvasive prenatal screening for expanded chromosome disease syndromes. Genet Med. 2019 Sep;21(9):1998-2006.

20 Salomon LJ, Alfirevic Z, Bilardo CM, Chalouhi GE, Ghi T, Kagan KO, et al. ISUOG practice guidelines: performance of first-trimester fetal ultrasound scan. Ultrasound $\mathrm{Ob}$ stet Gynecol. 2013 Jan;41(1):102-13.
21 Juneau K, Bogard PE, Huang S, Mohseni M, Wang ET, Ryvkin P, et al. Microarray-based cell-free DNA analysis improves noninvasive prenatal testing. Fetal Diagn Ther. 2014; 36(4):282-6.

22 Sparks AB, Wang ET, Struble CA, Barrett W, Stokowski R, McBride C, et al. Selective analysis of cell-free DNA in maternal blood for evaluation of fetal trisomy. Prenat Diagn. 2012 Jan 6;32(1):3-9.

23 Sparks AB, Struble CA, Wang ET, Song K, Oliphant A. Noninvasive prenatal detection and selective analysis of cell-free DNA obtained from maternal blood: evaluation for trisomy 21 and trisomy 18. Am J Obstet Gynecol. 2012 Apr;206(4):319-9.

24 S2k-Leitlinie Humangenetische Diagnostik und Genetische Beratung. Med Genet. 2018; 30:469-522.

25 Bunnell M, Zhang C, Lee C, Bianchi DW, Wilkins-Haug L. Confined placental mosaicism for 22q11.2 deletion as the etiology for discordant positive NIPT results. Prenat Diagn. 2017 Apr;37(4):416-9.

26 Wapner RJ, Babiarz JE, Levy B, Stosic M, Zimmermann B, Sigurjonsson S, et al. Expanding the scope of noninvasive prenatal testing: detection of fetal microdeletion syndromes. Am J Obstet Gynecol. 2015 Mar;212(3):332-9.

27 Ravi H, McNeill G, Goel S, Meltzer SD, Hunkapiller N, Ryan A, et al. Validation of a SNP-based non-invasive prenatal test to detect the fetal 22q11.2 deletion in maternal plasma samples. PLoS One. 2018;13(2): e0193476.

28 Neofytou MC, Tsangaras K, Kypri E, Loizides C, Ioannides M, Achilleos A, et al. Targeted capture enrichment assay for non-invasive prenatal testing of large and small size subchromosomal deletions and duplications. PLoS One. 2017;12(2):e0171319.

29 Koumbaris G, Kypri E, Tsangaras K, Achilleos A, Mina P, Neofytou M, et al. Cell-free DNA analysis of targeted genomic regions in maternal plasma for non-invasive prenatal testing of trisomy 21 , trisomy 18 , trisomy 13 , and fetal sex. Clin Chem. 2016 Jun;62(6):84855.
30 Helgeson J, Wardrop J, Boomer T, Almasri E, Paxton WB, Saldivar JS, et al. Clinical outcome of subchromosomal events detected by whole-genome noninvasive prenatal testing. Prenat Diagn. 2015 Oct 1;35(10):999-1004.

31 Gross SJ, Ryan A, Benn P. Noninvasive prenatal testing for $22 \mathrm{q} 11.2$ deletion syndrome: deeper sequencing increases the positive predictive value. Am J Obstet Gynecol. 2015;213: 254-255.

32 Pescia G, Guex N, Iseli C, Brennan L, Osteras $\mathrm{M}$, Xenarios I, et al. Cell-free DNA testing of an extended range of chromosomal anomalies: clinical experience with 6,388 consecutive cases. Genet Med. 2017 Feb;19(2):16975.

33 Wang Y, Li Y, Chen Y, Zhou R, Sang Z, Meng $\mathrm{L}$, et al. Systematic analysis of copy-number variations associated with early pregnancy loss. Ultrasound Obstet Gynecol. 2020 Jan; 55(1):96-104.

34 Benn P, Borrell A, Chiu RW, Cuckle H, Dugoff L, Faas B, et al. Position statement from the Chromosome Abnormality Screening Committee on behalf of the Board of the International Society for prenatal diagnosis. Prenat Diagn. 2015;35:725-34.

35 Bassett AS, McDonald-McGinn DM, Devriendt K, Digilio MC, Goldenberg P, Habel A, et al. Practical guidelines for managing patients with 22q11.2 deletion syndrome. J Pediatr. 2011 Aug;159(2):332-9.e1.

36 Cheung EN, George SR, Andrade DM, Chow EW, Silversides CK, Bassett AS. Neonatal hypocalcemia, neonatal seizures, and intellectual disability in 22q11.2 deletion syndrome. Genet Med. 2014 Jan;16(1):40-4.

37 Eicher PS, McDonald-Mcginn DM, Fox CA, Driscoll DA, Emanuel BS, Zackai EH. Dysphagia in children with a 22q11.2 deletion: unusual pattern found on modified barium swallow. J Pediatr. 2000 Aug;137(2):158-64. 